\title{
A Conceptual Design Model for High Performance Hotspot Network Infrastructure (GRID WLAN)
}

\author{
Udeze Chidiebele. C, Okafor Kennedy .C \\ R \& D Department, Electronics Development Institute \\ (FMST-NASENI), Awka, Nigeria.
}

\begin{abstract}
The emergence of wireless networking technologies for large enterprises, operators (service providers), small-medium organizations, has made hotspot solutions for metropolitan area networks (MAN), last mile wireless connectivity, mobile broadband solutions, IP-based cellular phones (VOIP) and other event-based wireless solutions in very high demand. Wireless radio gateways (routers and access points) with its wide-spread deployment has made $\mathrm{Wi}-\mathrm{Fi}$ an integral part of today's hotspot access technology in organizational models. Despite its role in affecting performance for mobility market segments, previous research has focused on media access control (MAC) protocol techniques , carrier sense multiple access with collision avoidance (CSMA/CA), fair scheduling, and other traffic improvement methodologies without detailed consideration for virtual switch partitioning for load balancing, reliable fragmentation capacities, buffer size dependencies, load effects, queuing disciplines as well as hotspot access control framework. This paper proposes a conceptual high performance hotspot solution (GRID WLAN) and through simulations with OPNET modeler, presents efficient performance metrics for its deployment. Considering the GRID WLAN access points in context, the results shows that with the design model, a careful selection of buffer sizes, fragmentation threshold, network management framework with network load intensity will guarantee an efficient hotspot solution.
\end{abstract}

Keywords: Hotspot solutions, IP-based cellular phones, Buffer size dependencies, GRID WLAN.

\section{INTRODUCTION}

The mobility market segments has made modern computing very attractive as such creating a platform for application developers INTEGRATE solutions in laptops, cloud computing environments as well as mobile devices. In this research, a conceptual high performance hotspot solution termed GRID WLAN is shown in Fig. 1. Anyone with a PC, notebook, mobile phone or PDA that is Wi-Fi-enabled can use the proposed GRID WLAN hotspot services.

From the user perspective, the network can be selected, browser launched and the user signs up by entering an authentication ID or by using a signed credit card for access control. The network comprises the infrastructure gateway (ADSL modem and GRID WLAN Gateway), Hypervisor layer (Virtualization firmware), GRID WLAN switch/load balancer, GRID WLAN Access points (AP1.......APn), Management framework, Wimax base station, VOIP, and hotspot nodes.

The network model adopted in this research is an extended service set GRID WLAN mode. The APs establishes connections to other users who are directly connected to the hotspots. Basically, FTP traffic and HTTP traffic take

\author{
Prof. H. C Inyiama, Dr C. C. Okezie, \\ Electronics and Computer Engineering Department, \\ Nnamdi Azikiwe University, Awka, Nigeria
}

place in the GRID WLAN hotspot zone sites while the GRID WLAN gateway access point creates a gateway link to the IP cloud (internet). The scope of this paper is majorly on the capacity of the various buffer sizes, loads as well as fragmentation threshold of the GRID WLAN APs as the principal metrics of interest in the proposed GRID WLAN design. This paper defines capacity in this context to be the maximum number of simultaneous, bidirectional traffic flow that can be supported in the hotspot environment.

The paper is organized as follows: In section II, the literature review was discussed, the general system model and assumptions for GRID WLAN hotspot was presented in III. In IV, GRID WLAN mechanism is presented. Section V gives the simulation results to support our propositions. The paper ends with the conclusions and future directions.

\section{LITERATURE REVIEW}

A detailed study on various works on hotspot WLAN solutions was carried out. However, a close attention was given to the work in [1] which is the most related work. The authors focused on WLAN performance issues viz: tuning the physical layer related parameters, MAC protocol techniques, tuning the IEEE 802.11 parameters, fair scheduling, and Carrier Sense Multiple Access with Collision Avoidance (CSMA/CA) and other traffic improvement methodologies.

A good introduction to the 802.11 standard, followed by a performance study of both the Distributed Coordination Function (DCF) and Point Coordination Function (PCF) is presented in [2]; this study suggests that an IEEE 802.11 network may be able to carry traffic with time-bounded requirements using the PCF. In Point Coordination Function (PCF) mode, polling occurs with a point coordinator determining which station has the right to transmit [3]. In the DCF mode, a BSS operates as an ad-hoc network in which any station can communicate with any other station in the BSA without the intervention of a centralized access point (AP) by contending for a shared channel. All stations have equal priorities and hence an equal chance of getting the channel [3].

Basically, the current use of WLANs for Internet access from wireless stations is dominated by downlink TCP traffic; however, the optimal performance of WLAN setups have not been sufficiently taken into account for data transport over WLANs devices like APs. WLAN parameters can be fine tuned to optimize performance. This has not been addressed in the previous literature reviews [4], [5].This paper shall focus on metrics that will enhance performance in a hotspot setup as shown in our system model in Fig.1. 


\section{System Design AND Assumptions}

\section{A. System Model}

The high performance GRID WLAN hotspot solution is an extended service set wireless configuration shown in Fig. 1. It assumed that the GRID WLAN gateway is a composite powerful gateway combined with radio which acts as a complete hotspot. At the core of the proposed GRID WLAN is a switch load balancer (bridge network) which acts as a distribution medium for all indoor access points, wired LAN, outdoor hotspots nodes, wimax base station, back office server (management framework). This paper practically details the implementation methodology as well as discusses the GRID WLAN mechanism in the section IV.

\section{B. Design Goals}

In designing our proposed GRID WLAN hotspot solution,

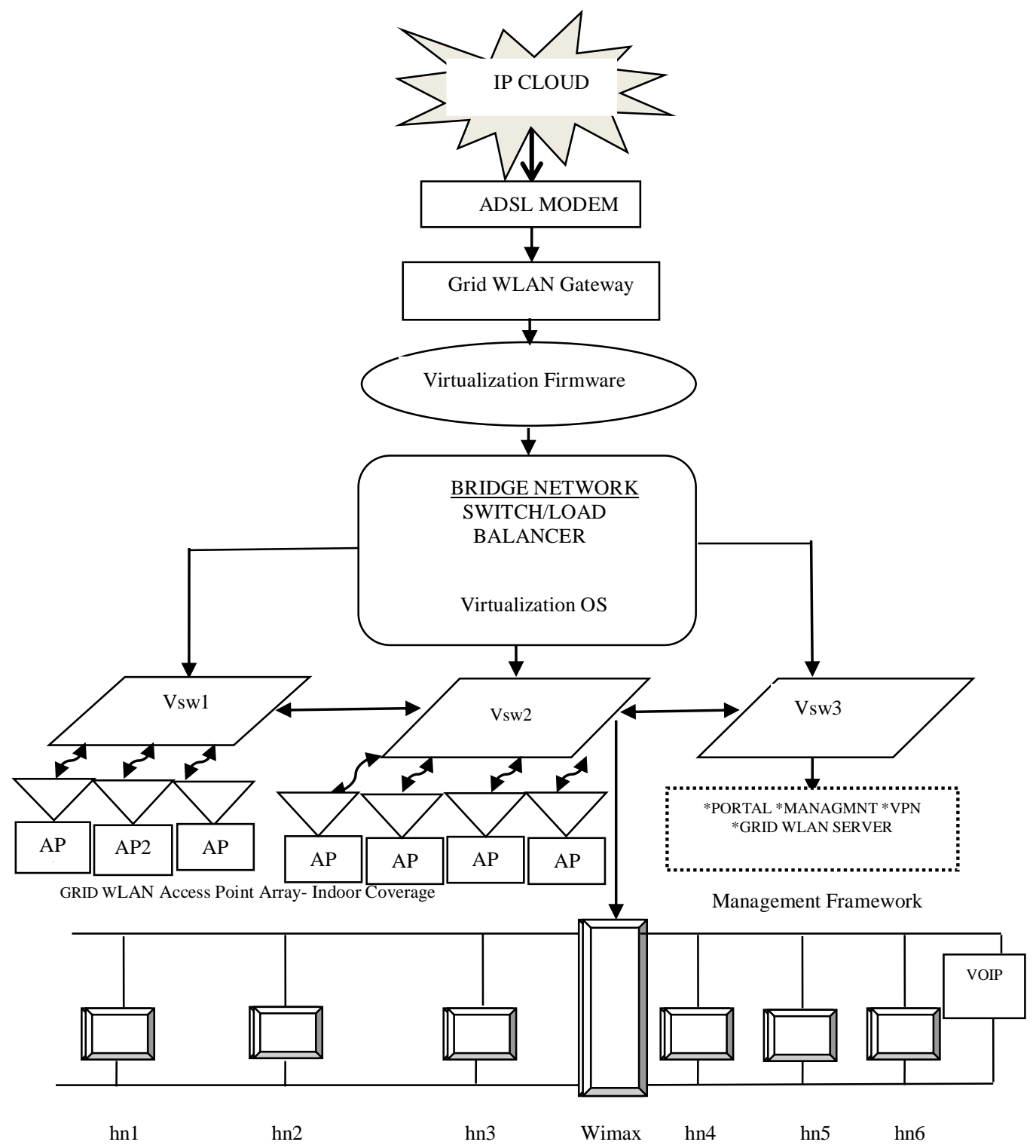

the main goals are to maintain throughput and to create a wireless hotspot solution that is based on robust network infrastructure, hence scalable, flexible and quick to deploy at anytime with less administrative overhead. Fig. 1 shows the proposed system model.

As shown in Fig 1, the core LAN switch with virtual OS connects the ADSL modem, management framework ,GRID WLAN access points (for indoor coverage site), the Wimax base station (outdoor coverage site), local hotspot nodes (hsn 1.....hns6), and VOIP. For our large hotspots including mesh nodes, the high powered GRID WLAN gateway will be capable of supporting over 500 simultaneous user sessions. In this context, we assume the GRID WLAN hotspot to be a combination of wired access points, Wimax base station, and indoor and outdoor mesh access point nodes dependent on the local site.

Figure 1. The proposed GRID WLAN Hotspot system model 


\section{Experimental Test Bed}

In this paper, to measure the system performance of the proposed GRID WLAN hotspot network infrastructure, OPNET Modeler [6] was used to achieve the objective. OPNET Modeler is a graphical network simulator mainly used for simulation of both wired and wireless communication networks and information systems. For our proposed test bed, the infrastructure components include:

i. 5 Hotspots nodes for outdoor Coverage

ii. Bridge Switch/Load balancer with virtual OS (Extended service mode).

iii. 5 Access Points for indoor coverage

iv. $\quad 40$ WLAN client Stations

v. Management Server (Enterprise Red hart Server)

vi. Back office Applications (Network Monitoring,

Configuration, Authentication, Authorization and Accounting ) :

- Grid Control Management server for central network configuration \& Monitoring.

- Portal Server for Network Services and management.

- VPN tunnel Terminator server

In Fig 1, the proposed GRID WLAN Hotspot system model utilizes the infrastructure components outline above. A management server has the role authenticating and monitoring the overall network for efficient service delivery. The extended service mode of the bridge switch enables both indoor and outdoor connectivity. The virtual switching by the switch bridge interfaces the management server, hotspot nodes, access points and client stations in our context as shown in Fig. 2a. The Access points were setup in the various subnets with the client base stations (Fig. 2b). The bridge switch is a speed redundancy layer supporting virtualization and load balancing. Access to the IP internet cloud is completed by the gateway and modem. The network model, node model and process models were accomplished in our test bed using OPNET modeller. After setting up the model, a simulation run was carried out to generate our graphical plots shown in this work (Fig 3). Also, a consistency test carried out shows that the design model is stable and consistent before the simulation execution.

\section{DISCUSSION}

From Fig. 1, the GRID WLAN hotspot solution employs the concept of mesh routing to create a self configurable, centrally controlled hotspot solution. Every GRID WLAN access point (AP) is capable of relaying traffic coming from any of its neighbours. The mesh network reconfigures itself when a node is added or removed. By adding a GRID WLAN access point (AP), the wireless network range is expanded. Owing to the self-reconfigurable mesh routing algorithms, the network has no point of failure, hence, a reliable wireless platform for connectivity

The GRID WLAN gateway router supports the following functionalities: Automatic Configuration, Bandwidth Control/Optimization, Firewall, Multiple Services, and Virus Checking with Extensive Access list control.

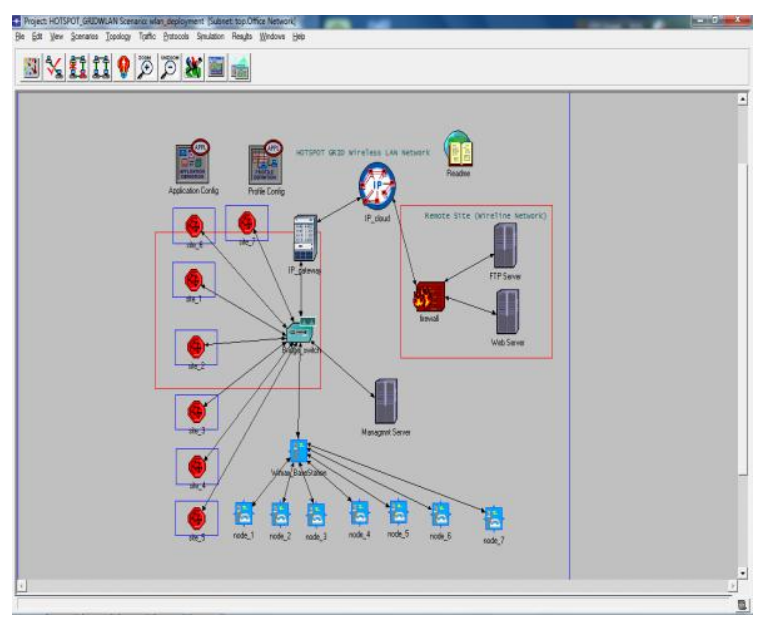

Figure 2. GRID WLAN testbed Implementation with subnetted clients

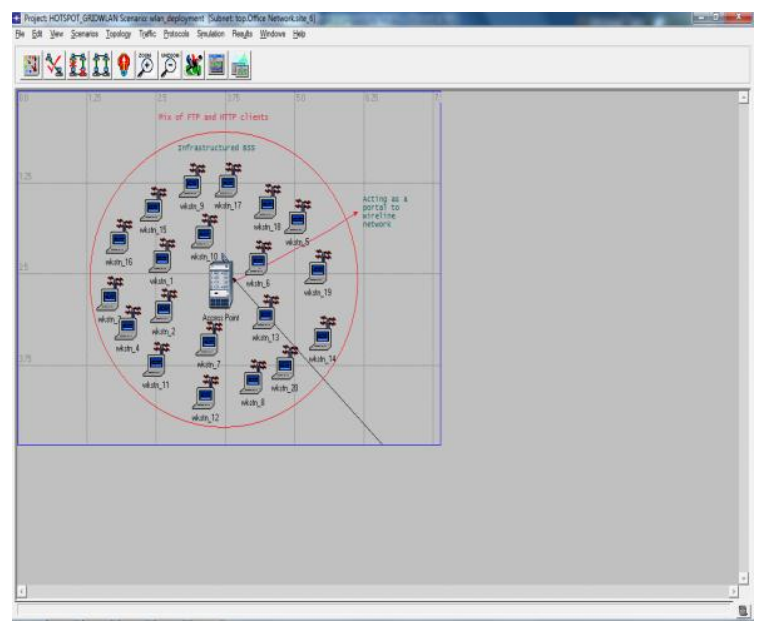

Figure 2b: GRID WLAN nodes

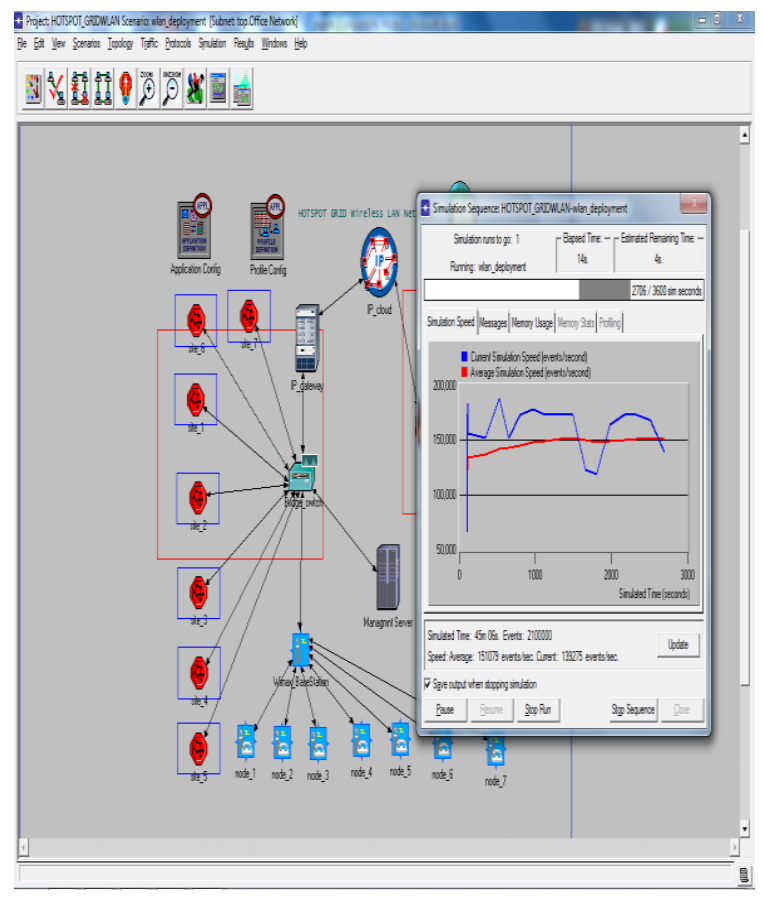

Figure 3. shows the GRID WLAN Simulation run 


\section{SimUlation PARAMETERS}

In this section, we provide simulation results that support section III. The GRID WLAN APs including the gateway router connects wireless network (nodes) to wired network to wired networks via the bridge switch as depicted in our model.

\section{A. Simulation Configurations}

We used OPNET modeler [6] was used to generate the parameters for various case scenarios in the simulations. Traffic attributes are listed in Table 1, while Table 2 show the GRID WLAN parameters used and the various load intensities from OPNET Modeler.

TABLE 2. GRID WLAN SIMULATION PARAMETER
TABLE 1. OPNET TRAFFIC ATTRIBUTES

\begin{tabular}{|c|l|l|}
\hline & \multicolumn{1}{|c|}{ CONFIGURATIONS } & \multicolumn{1}{c|}{ Values } \\
\hline 1. & $\begin{array}{l}\text { Simulation Duration for Each } \\
\text { Scenario }\end{array}$ & $60 \mathrm{mins}$ \\
\hline 2. & Link Propagation Delays & 0.5 usecs \\
\hline 3. & Switch Output Buffer & 100 packets \\
\hline 4. & Simulation Seed & 128 \\
\hline 5. & Update Interval & 50000 Events \\
\hline 6. & Simulation Kernel & Optimized \\
\hline
\end{tabular}

\begin{tabular}{|c|c|c|c|c|c|c|}
\hline $\begin{array}{c}\text { WLAN } \\
\text { PARAMETERS }\end{array}$ & $\begin{array}{l}5 \text { Sources } \\
\text { WLAN } \\
\text { BUFFER- } \\
64 \mathrm{~K}\end{array}$ & $\begin{array}{l}10 \text { Sources } \\
\text { WLAN } \\
\text { BUFFER- } \\
128 \mathrm{~K}\end{array}$ & $\begin{array}{l}15 \text { Sources } \\
\text { WLAN } \\
\text { BUFFER- } \\
256 \mathrm{~K}\end{array}$ & $\begin{array}{l}20 \text { Sources } \\
\text { WLAN } \\
\text { BUFFER- } \\
512 \mathrm{~K}\end{array}$ & $\begin{array}{l}30 \text { Sources } \\
\text { WLAN } \\
\text { BUFFER- } \\
1024 \mathrm{~K}\end{array}$ & $\begin{array}{l}\text { 40 Sources } \\
\text { WLAN } \\
\text { BUFFER- } \\
2048 \mathrm{~K}\end{array}$ \\
\hline $\begin{array}{l}\text { RTS-Threshold } \\
\text { (Bytes) }\end{array}$ & 256 Bytes & 256Bytes & 256Bytes & 256Bytes & 256Bytes & 256Bytes \\
\hline $\begin{array}{c}\text { Fragmentation } \\
\text { Threshold (Bytes) }\end{array}$ & 256 Bytes & 512Bytes & 1024Bytes & 2048Bytes & 4096Bytes & 8192Bytes \\
\hline Data Rate (bps) & 54 & 54 & 54 & 54 & 54 & 54 \\
\hline $\begin{array}{c}\text { Physical } \\
\text { Characteristics }\end{array}$ & DSSS (D3S) & DSSS & DSSS & DSSS & DSSS (D3S) & DSSS (D3S) \\
\hline $\begin{array}{c}\text { Packet-Reception } \\
\text { Power } \\
\text { Threshold(W) }\end{array}$ & $7.33 \mathrm{e}-14$ & $7.33 \mathrm{e}-14$ & $7.33 \mathrm{e}-14$ & $7.33 \mathrm{e}-14$ & $7.33 \mathrm{e}-14$ & $7.33 \mathrm{e}-14$ \\
\hline Short Retry Limit & 7 & 7 & 7 & 7 & 7 & 7 \\
\hline Long Retry Limit & 4 & 4 & 4 & 4 & 4 & 4 \\
\hline AP Functionality & Active & Active & Active & Active & Active & Active \\
\hline Buffer Size (bits) & $64 \mathrm{~K}$ & $128 \mathrm{k}$ & $256 \mathrm{k}$ & $512 \mathrm{k}$ & $1024 \mathrm{~K}$ & $2048 \mathrm{~K}$ \\
\hline $\begin{array}{l}\text { Max-Receive } \\
\text { Lifetime (Sec) }\end{array}$ & 0.5 & 0.5 & 0.5 & 0.5 & 0.5 & 0.5 \\
\hline $\begin{array}{c}\text { Large-Packet } \\
\text { Processing }\end{array}$ & Drop & Drop & Drop & Drop & Drop & Drop \\
\hline
\end{tabular}

TABLE $3 . \quad$ GRID WLAN SCENARIO TABLE

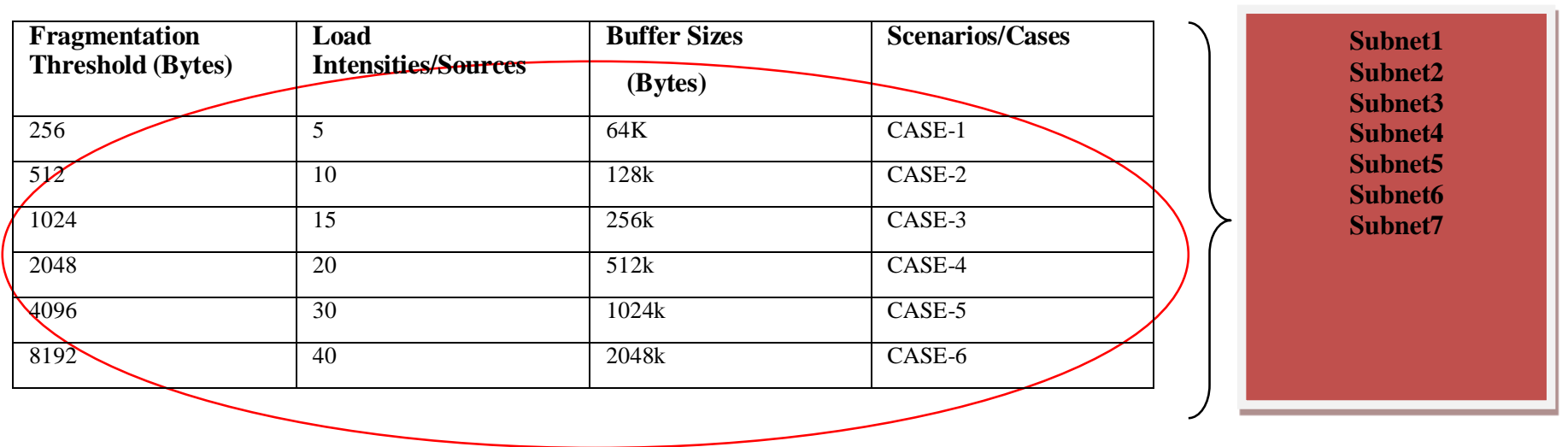

\section{B. Performance Evaluation}

The simulation was run accordingly and the statistics collected for various cases and analyzed. As claimed before, the proposed GRID WLAN hotspot is a high performance solution that is robust and scalable. The paper used a casebased scenario for various load intensities as well as 
fragmentation threshold and buffer sizes as depicted in Table 3.0

To demonstrate the effects of Fragmentation Threshold, we employed the 5 node simulation scenarios with combinations of values for Fragmentation Threshold and RTS as well as other parameters. After the first case simulations, the throughput was collected for analysis. The throughput is the bit rate sent to the higher layer. It represents the rate of data successfully received from other stations. At the offered load from one node source to five node sources, it was shown that for a buffer size of 64 bytes for the FTP and HTTP traffic, the throughput had a slight deviation at $89 \%$ showing a significant network performance.

Fig 7: shows the media access delay for offered Load time against fragmentation threshold Fragmentation threshold is an important parameter that affects WLAN performance. It is used to improve the WLAN performance when the media error rate is high.

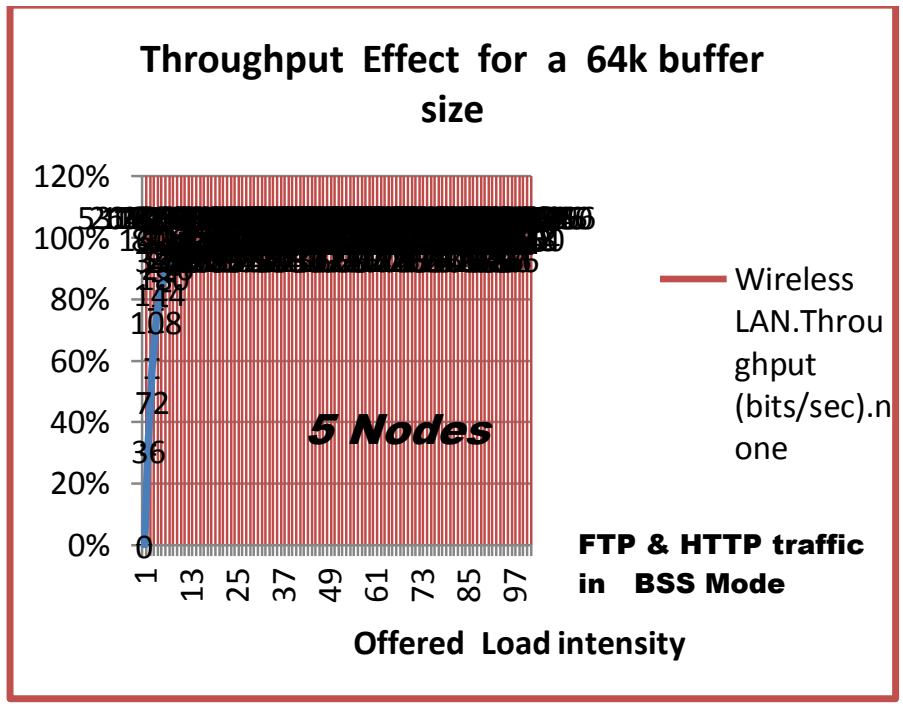

Figure 6: A plot of Offered Load Versus Processed Throughput for a $64 \mathrm{k}$ buffer size

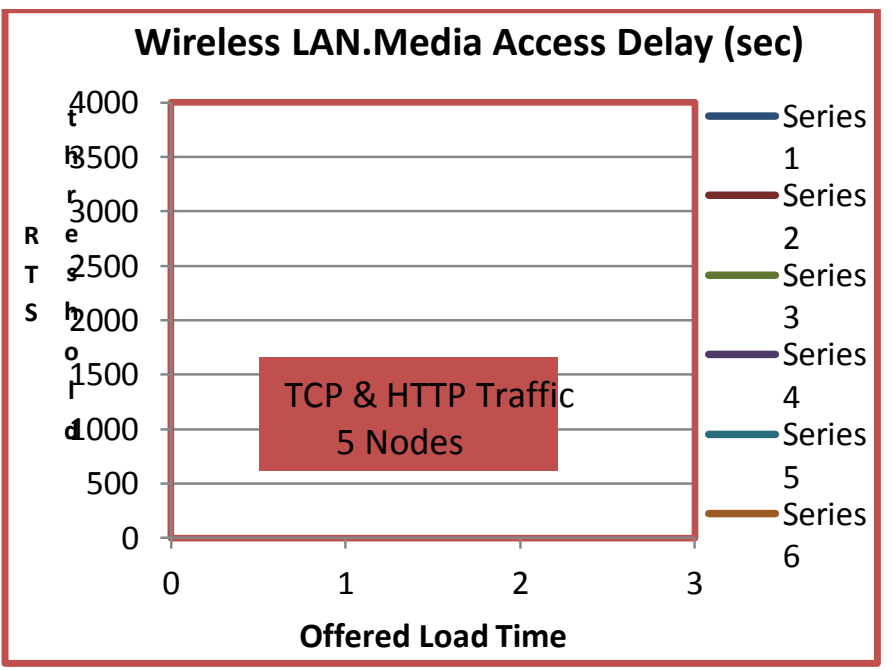

Figure 7: Offered Load time against fragmentation threshold
From Fig. 7, the effect of Fragmentation threshold on the WLAN performance was seen to be insignificant considering the offered load times. This could result from the selected threshold in the APs and client machines. For the first five simulation scenarios, parameters for Fragmentation Threshold are listed in Table 2. The simulation results indicate that for low bit error rates $(2 \times 10-5)$, various fragmentation thresholds (256 bytes, 512 bytes, or no fragmentation limit ) have no significant effect on the WLAN performance.

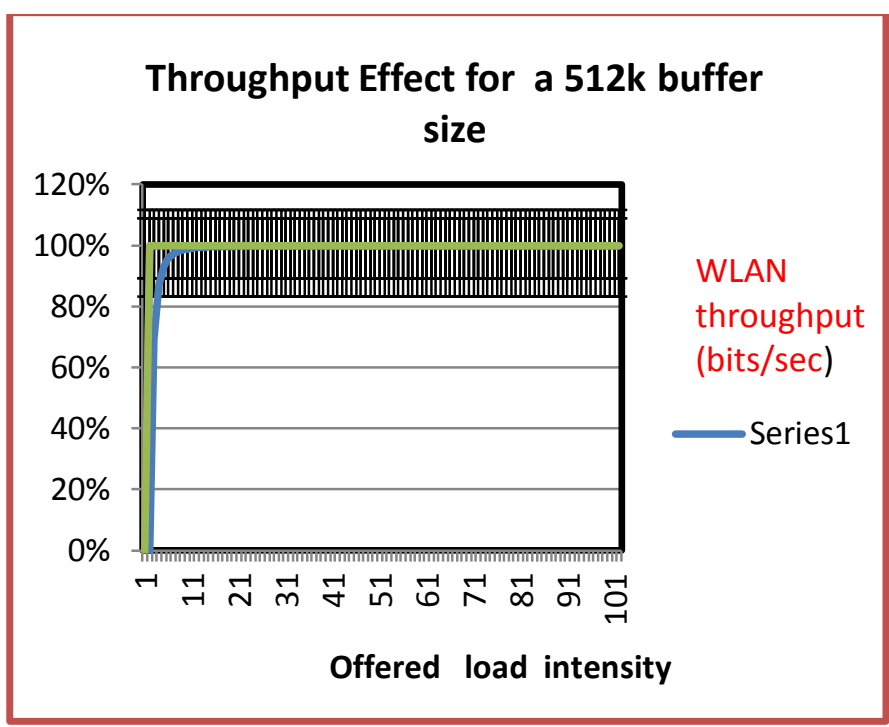

Figure 8: A plot of Offered Load Versus Processed Throughput for a $512 \mathrm{k}$ buffer size

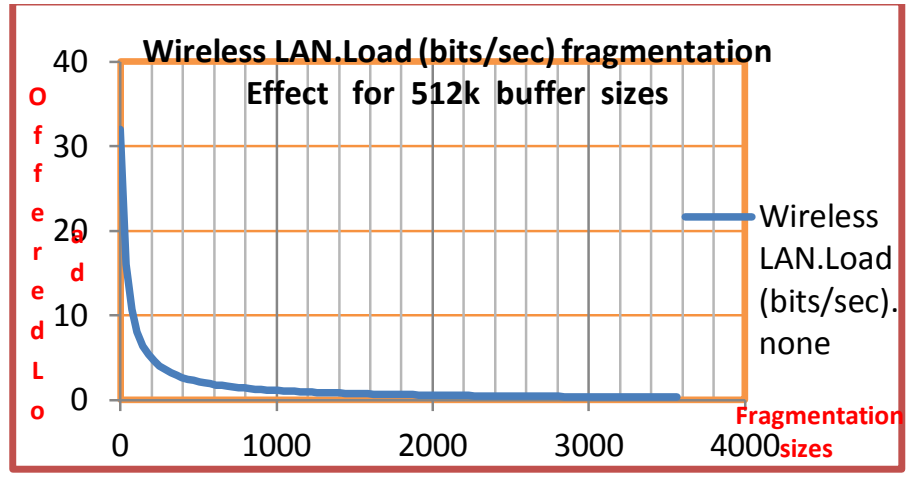

Figure 9: A plot of WLAN Load Against fragmentation threshold for a $512 \mathrm{k}$ buffer size.

Fig. 8 shows the throughput effect on offered load intensity for a $512 \mathrm{k}$ buffer size. From one to five workstations, there was a gradual exponential response until at a saturation point of about $87 \%$, then it now begin to maintain a constant throughput with additional loads. For, every network, traffic optimization can only be achieved when the network throughput is significantly acceptable or high.

The simulation results in Fig. 9 illustrates that with an adaptive back-off mechanism, the offered load between WLAN stations can be greatly decreased with increase in the 
fragmentation sizes while throughput can still maintain the same or achieve a slightly higher value. The reduction of WLAN load is important for power reduction in wireless devices. The Fragmentation threshold parameters used for the simulation run indicate that when the bit error rate.

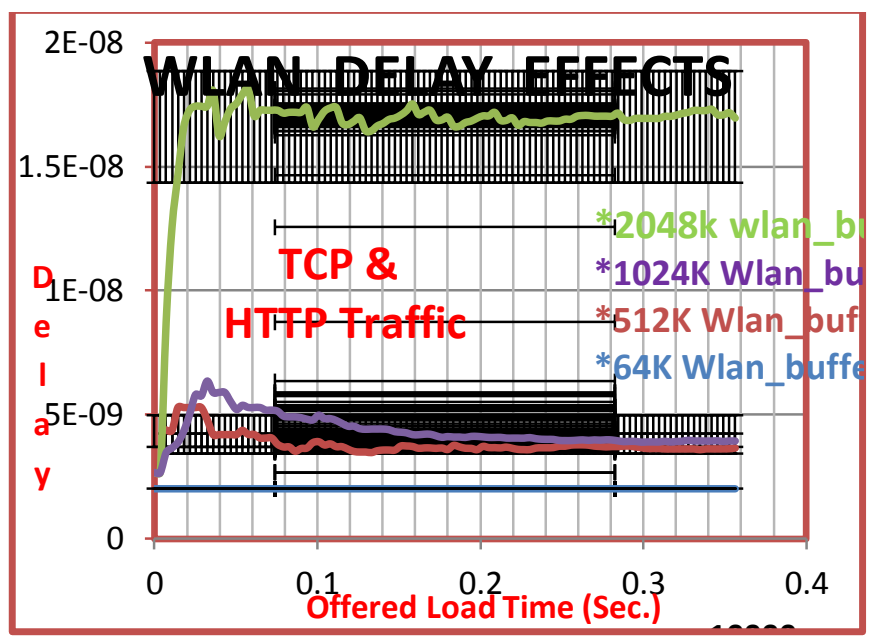

Figure 10: A Plot Of WLAN Delay Effects For 64k,512k,1024k,\&2048k

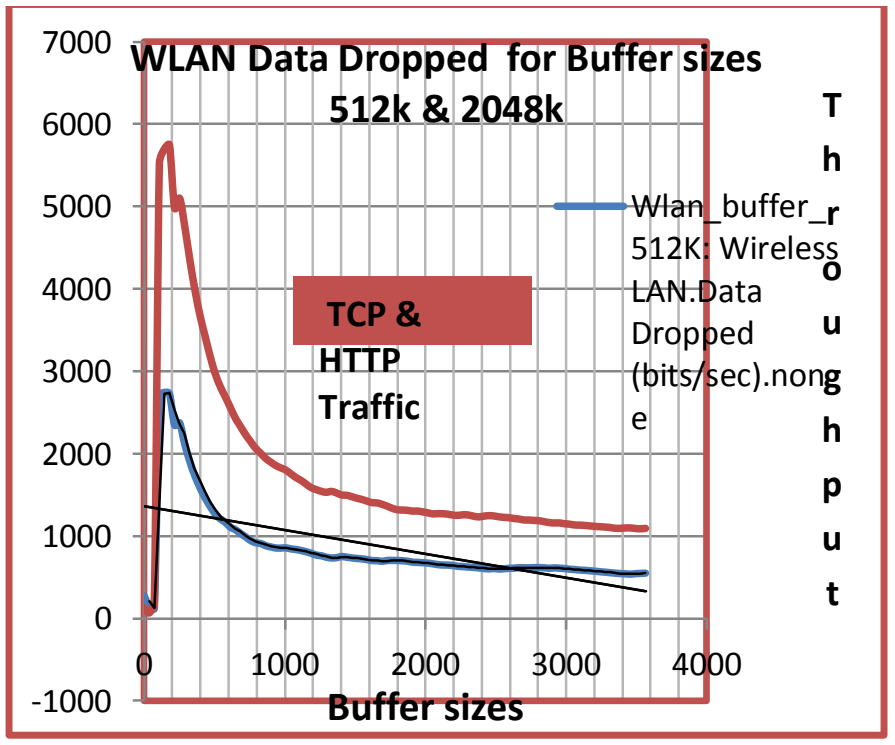

Figure 11: A plot of WLAN Data Dropped for 512K \& 2048K buffer Sizes

During the simulations, the media access delay i $\mathrm{n}$ the four cases above was collected. The media access delay is the sum of queue and contention delays of data packets received by the WLAN MAC layer from the higher layer. For each packet, the delay is recorded when the packet is sent to the physical layer.

The simulation results (Fig.10) indicate that smaller buffer size values can decrease the average media access delay, while larger buffer sizes increases average media delay. Also the average media access delay is increased with increase in load intensities. This shows that performance of a wireless LAN is reduced with an increase in load intensity even when the buffer size is as well increased.
Fig. 11 shows simulated DCF throughput as a function of offered load for 5-40 wireless stations. Offered load in this context depicts the WLAN client nodes. First, we note that for the 10 and 20 stations, throughput is higher for $2048 \mathrm{k}$ buffer size compared with $512 \mathrm{k}$ buffer size. As offered load is increased, throughput grows linearly until a saturation point at which throughput ceases to increase; in fact, it decline. When the number of stations is large, throughput decrease and hence packet drop is inevitable. The drop in peak and saturation throughput as a function of the number of wireless stations is shown in Fig. 11.

At a moderate load of 16-20 wireless nodes, peak throughput decreases for buffer sizes 512k-2048k. Hence, for efficient network performance, the number of workstations should be carefully chosen and the AP device properly selected for high throughput in all cases.

\section{CONCLUSION}

This research has presented a conceptual GRID WLAN hotspot model with performance analysis which can be further expanded to address the security challenges of the Wimax base station. The work investigates the effects of GRID WLAN APs buffer size distributions with respect to load intensities as well as fragmentation capacities. The analysis and measurement results from OPNET simulation shows that a careful selection of buffer sizes, fragmentation thresholds in GRID WLAN hotspot model can help to optimize network performance. Although some of these results are characteristic of the designed network, I can conclude that the GRID WLAN hotspot network performance is mainly based on the simplification of the traffic in addition to mobility and the buffer sizes.

\section{REFERENCES}

[1] Jiaqing Song and Ljiljana Trajkovic: Enhancements and performance evaluation of wireless local area networks.

[2] B. P. Crow, I.Widjaja, J. G. Kim, and P. T. Sakai. IEEE 802.11 wireless local area networks.IEEE communications Magazine, September 1997.

[3] S. Khurana, A. Kahol, S. K. S. Gupta and P. K. Srimani :Performance evaluation of distributed co-ordination function for IEEE 802.11 Wireless LAN protocol in presence of mobile and hidden terminals (Unpublished).

[4] LAN MAN Standards Committee of the IEE Computer Society, "Part 11: wireless LAN medium access control (MAC) and physical layer (PHY) specifications," ANSI/ IEEE Standard 802.11, 1999 Edition.

[5] L. bononi, m. conti, and L. donatiello, "design and performance evaluation of a distributed contention control (dcc) mechanism for ieee 802.11 wireless local area networks," in proceedings of first Acm international workshop.

[6] C.-H. Ng, J. Chow, and Lj. Trajkovic, "Performance evaluation of the TCP over WLAN 802.11 with the snoop performance enhancing proxy," OPNETWORK 2002, Washington, DC, Aug. 2002.

[7] .S. A. Bawazir, S. H. Al-Sharaeh, "Performance of infrastructure mode wireless LAN access network based on OPNET Simulator" Department of Computer Science, Normal, AL 35762,USA

[8] P. Jarmo, "OPNET - Network Simulator", seminar presented at VTT Technical Research Centre of Finland, March, 2006

[9] J.Singh, Quality Of service in wireless Lan Using OPNET Modeller, Available at http://dspace.thapar.edu.

[10] M. S. Gast, 802.11 Wireless networks: the definitive guides, O’Reilly, 2002. 
[11] A. Banchs, "Analysis of the delay distribution in 802.11 DCF: A step towards delay guarantees in WLANs," LNCS, vol. 3266, Sept. 2004, pp. 64-73.

[12] IEEE 802.11e/D13.0, Part 11, "Wireless LAN medium access control (MAC) and physical layer (PHY) specifications: medium access Control (MAC) enhancements for Quality of Service (QoS)," draft supp. to IEEE 802.11 std., Jan. 2005.

[13] R. Litjens et al., "Performance analysis of wireless LANs: an integrated packet/flow level approach," Proc. 18th Int'l. Teletraffic Cong., Sept. 2003, pp. 931-40.

[14] B. Liu, Z. Liu, and D. Towsley. On the capacity of hybrid wireless networks. In Proc. IEEE INFOCOM '03, pages 1543-1552, 2003.
[15] T. Hansen, P. Yalamanchili and H-W. Braun, "Wireless measurement and analysis on HPWREN", Proceedings of Passive and Active Measurement Workshop, Fort Collins, Co, pp. 222-229, March 2002.

[16] M. H.Manshaei and T. T. INRIA, "Simulation-based performance analysis of 802.11a Wireless LAN". Route des Lucioles, BP-93, 06902 Sophia-Antipolis Cedex, France ,2004.

[17] J. Song and L. Trajkovic, "Enhancements and performance evaluation of wireless LAN". Communication Networks Laboratory Simon Fraser University, Burnaby, BC, Canada.

[18] Kaur1, S. Vijay, S.C. Gupta, "Performance analysis and enhancement of IEEE 802.11 wireless LAN". Global Journal of Computer Science and Technology Vol. 9 Issue 5 (Ver 2.0),130 ,January 2010 . 